\title{
Role of Three Dimensional Doppler Ultrasonography And Leukemia Inhibitory Factor From Endometrial Secretion In Predicting Clinical Pregnancy In IVF Treatment
}

Ivan Sini ( $\sim$ ivansini@irsi-bunda.org )

Indonesian Reproductive Science Institute https://orcid.org/0000-0002-5364-0381

Nining Handayani

Indonesian Reproductive Science Institute

Alida MD, Ph.D Harahap

University of Indonesia

Arief Boediono

IPB University

Budi Wiweko

University of Indonesia

Wachyu Hadisaputra

University of Indonesia

Soegiharto Soebijanto

University of Indonesia

Tri Aprilliana

IRSI Research and Training Centre

Arie Adrianus Polim

IRSI Research and Training Centre

Aryando Pradana

Morula IVF Jakarta Clinic

\section{Research Article}

Keywords: Endometrial receptivity, Leukemia Inhibitory factor, power Doppler ultrasound, sub-endometrial vascularization

Posted Date: September 17th, 2021

DOl: https://doi.org/10.21203/rs.3.rs-831597/v1 
License: (c) (i) This work is licensed under a Creative Commons Attribution 4.0 International License. Read Full License 


\section{Abstract}

\section{Purpose}

This study aimed to evaluate the potential synergistic role of three-dimensional power Doppler angiography ultrasound and the expression of Leukemia Inhibitory factor (LIF) protein in predicting the endometrial receptivity of fresh In Vitro Fertilization (IVF) cycles.

\section{Materials and methods}

This prospective study involved 29 good prognosis women who underwent fresh IVF cycles with fresh blastocysts transfer. Serial measurements of sub-endometrial parameters via power Doppler angiography including vascularity index ( $\mathrm{VI})$, flow index $(\mathrm{FI})$, and vascularization flow index (VFI) were conducted consecutively on the day of oocyte maturation trigger, ovum pick-up, and blastocyst transfer. Aspiration of endometrial secretion was performed on the day of embryo transfer.

\section{Results}

After performing multiple logistic regression analyses, the mean index of VI on the trigger day and LIF protein concentration at the window of implantation was significantly higher in clinically pregnant women than that of the non-pregnant women $(p<0.05)$. The area under the curve (AUC) of LIF concentration was shown to have a powerful predictive value to forecast clinical pregnancy $(0.884, p$ $<0.05$ ), with a cut-off value of $713 \mathrm{pg} / \mathrm{mL}$. Likewise, $\mathrm{VI}$ index on the trigger day was adequate to become a predictor for the pregnancy event (AUC 0.803 , the cut-off value $>5.381, p<0.05$ ). A combination of both variables utilizing the cut-off value was adequate for the differentiation of pregnant and non-pregnant cases $(p<0.05)$.

\section{Conclusion}

$\mathrm{VI}$ index assessed on trigger day and the expression of LIF protein concentration at the window of implantation provided sufficient information to predict endometrial receptivity.

\section{Introduction}

Successful implantation reflects an excellent bi-directional communication and interaction between embryos and maternal endometrium at the receptive phase through a molecular pathway. Even though embryo viability has been considered as the prominent factor contributing to successful implantation, endometrial receptivity is also critical to provide a suitable environment that allows a blastocyst to implant. Endometrial receptivity is defined as a period of endometrial maturation characterized by histological changes that are driven by the consecutive actions of steroid hormones, estradiol and progesterone, in the mid-luteal phase [1]. Despite the various clinical and laboratory improvements in InVitro Fertilization (IVF) treatment for infertile couples, precise markers for measuring endometrial receptivity remain a focus of extensive research. A comprehensive investigation on the multi-omics of 
endometrium (i.e., genomics, proteomics, secretomes, metabolomics), cytokines profile, and ultrasound marker including Doppler signals have long been explored, but none of them have yielded a gold standard diagnostic test [2].

A possible non-invasive indicator of endometrial receptivity in IVF is through the ultrasound assessment of endometrial and sub-endometrial vascularization. Adequate endometrial blood flow and perfusion are prerequisites of endometrial preparation for embryo implantation and are believed to reflect endometrial receptivity. Kupesic and Coworkers indicated that a lack of sub-endometrial perfusion during periovulation was associated with failure of implantation in IVF cycles [3]. Nonetheless, despite the promising results of the three-dimensional power Doppler angiography in various studies $[4,5]$, correlating this marker with implantation potential remains controversial $[2,6,7]$.

On the other hand, Leukemia inhibitory factor (LIF) is a well-recognized endometrial cytokine that possesses an important role in the event of implantation [8, 9]. LIF is a pleiotropic factor of the interleukin- 6 family which is prominently transcribed and expressed, during the early secretory phase in both the luminal and glandular epithelium [8]. Its interactions with other proteins and factors are essential to promote trophoblastic cell motility. An initial study conducted by Stewart and colleagues has found that in LIF-knockout mice, LIF played a significant role in implantation [10].

In humans, altered expression of LIF in infertile women with unexplained infertility has been demonstrated [11], followed by a 2017 study, which found that women with recurrent implantation failure might have an initial dysregulation of the immune and inflammatory response of LIF, progestagenassociated endometrial protein (PAEP), and interleukin-6 signal transducer (IL6ST) [12]. In concordance with those findings, a blastocyst has been proven to express LIF receptor (LIFR) [13], providing evidence for the important regulatory function of LIF in the implantation process. It is interesting to note that previous investigations of LIF in relation to implantation have been focused more on infertile women versus fertile women $[11,12,14]$, while the presence of LIF in endometrial cavity during peri-implantation of a fresh IVF cycle has yet been studied intensively. The objective of this study is to understand the significance of three-dimensional Doppler ultrasound of the sub-endometrial vascularity and LIF concentration at the implantation window of a fresh IVF cycle in predicting a clinical pregnancy.

\section{Material And Method}

\subsection{Patients}

The subjects recruited were infertile patients who underwent their first IVF cycle at Morula IVF Clinic Jakarta, Indonesia between January 2019 - December 2020. The patients were counseled to participate in the study at the beginning of the stimulation treatment. The inclusion criteria included regular menstrual cycle, no uterine abnormality, and normal ovarian reserve. The exclusion criteria were women with uterine pathologies (i.e., uterine myoma, endometrial polyp, endometriosis, and adenomyosis) and those with a history of uterine surgery (i.e., myomectomy, adenomyosis resection, and curette operation of more than 
three times). The sample size was calculated to have a sufficient power of $80 \%(n=28)$ to detect the differences between pregnant and non-pregnant women.

\subsection{Assisted reproductive treatment}

All subjects were subjected to ovarian stimulation antagonist protocol. Gonadotropin recombinant FSH (rFSH, Gonal F®, Merck Serono) or a combination of recombinant FSH and recombinant $\mathrm{LH}(\mathrm{rFSH} / \mathrm{rLH}$, Pergoveris $\AA$, Merck Serono) or urinary Human Menopausal Gonadotropin (UHMG) were administered daily starting on day 2 or 3 of the menstrual cycle. The initial dose ranged between 150 IU to $300 \mathrm{IU}$ per day. Injection of $0.25 \mathrm{mg} \mathrm{GnRH}$ antagonists (Cetrotide®, Merck KGaA) was commenced on day 5 of the stimulation. Recombinant Human Chorionic Gonadotropin (rHCG $250 \mathrm{mcg}$, equivalent to 6,500 IU, Ovidrel ${ }^{\circledR}$, Merck Serono) was given as the final oocyte maturation injection when three dominant follicles had reached $18 \mathrm{~mm}$ in diameter. Oocyte retrieval procedure was performed 36 hours afterward under mild sedation. Intracytoplasmic Sperm Injection (ICSI) was performed in sperm factor infertility and teratozoospermic cases. All embryos were cultured to the blastocyst stage. Good quality blastocysts were evaluated on day 5 after the ICSI procedure according to the Gardner criteria (grade 3-5 of blastocoel expansion with a combined score of inner cells mass and trophoblast cells of $A A, A B$, or BA) [15]. An embryo transfer procedure of maximum two good blastocysts was performed with a soft catheter (K-JET7019-ET, Cook, USA). All patients received progesterone for luteal support starting on the OPU day until hCG testing (vaginal progesterone: 200 mg Utrogestan ${ }^{\circledR}$ (Besins Healthcare, Belgium)/12 hours and 90 mg Crinone ${ }^{\circledR}$ (Merck, Serono)/day). Positive Beta HCG result was confirmed using transvaginal ultrasound four weeks after OPU.

\subsection{Three dimensional Doppler ultrasound measurement}

Transvaginal ultrasound scans were utilized to evaluate follicular and endometrial development. Three dimensional Doppler assessments were performed during the day of rHCG injection, oocyte retrieval, and embryo transfer. Histogram data were captured with built-in VOCAL (Virtual Organ Computer-aided Analysis) software in all ultrasound machines used for this study (Voluson S10, General Electric, USA). The sub-endometrium measurement was taken in the sagittal plane and indices of Vascularization Index, Flow Index, and Vascularization Flow Index were calculated automatically.

\subsection{Endometrial secretion collection}

Endometrial secretion was collected immediately before blastocyst transfer. The procedure was performed as described by previous studies $[16,17]$. As the patient was lying in a lithotomy position prior to embryo transfer, a speculum was inserted and the cervix was cleaned with saline. The sterile soft catheter was inserted into the uterine cavity through the guidance of an ultrasound scan. As the catheter 
tip reached the mid cavity, the endometrial secretion sample was aspirated with a $10 \mathrm{ml}$ syringe and flushed into a sterile tube up to $100 \mu \mathrm{L}$. All samples were then stored at $-20 \mathrm{C}$.

\subsection{LIF analysis}

Endometrial secretion samples were diluted with Phosphate Buffered Saline (PBS) solution up to a volume of $100 \mu \mathrm{L}$. Human LIF commercial kit for Sandwich enzyme-linked immunosorbent assay (ELISA) (Invitrogen/Thermo Fischer Scientific, US) was used according to the manufacturer's protocol to analyze the samples. Sensitivity of the ELISA kit was in the range of $<5-500 \mathrm{pg} / \mathrm{mL}$.

\subsection{Statistical analysis}

Statistical analyses were performed using the Statistics Package for Social Sciences (SPSS) version 20.0 (SPSS Inc, Chicago, IL, USA). All variables were evaluated for normal data distribution with the ShapiroWilk test. SQRT method was utilized to normalize non-normal data distribution. Numerical variable analysis was performed using T-test or Mann-Whitney test. Categorical variables were analyzed using Chi-squared tests. Multiple analysis using the backward logistic regression method was used to adjust potential confounders. The probability of $<0.05$ was considered statistically significant.

\section{Results}

The age of women in this study was relatively young with normal BMI range $\left(<25 \mathrm{~kg} / \mathrm{m}^{2}\right)$. Most participants had primary infertility with sperm factor and unexplained infertility as the most common causes of infertility followed by recurrent IUI failures. Clinical characteristics of the study subjects exhibited a normal hormonal profile signifying good prognosis (Table 1). All the women underwent the embryo transfer of good quality blastocyst(s). Eventually, 11 achieved pregnancy, while the remaining subjects $(n=18)$ did not get pregnant. The overall pregnancy rate in this study was $37.9 \%$. 
Table 1

Baseline, clinical characteristics, and embryology results of studied subjects

\begin{tabular}{|ll|}
\hline Parameters & Value $(\mathbf{n}=29)$ \\
\hline Baseline & \\
\hline Female age (years) & $31(27-40)$ \\
\hline Body mass index $\left(\mathrm{Kg} / \mathrm{m}^{2}\right)$ & $23.83 \pm 3.86$ \\
\hline Type of infertility & \\
\hline Primary infertility & $24(82.8 \%)$ \\
\hline Secondary infertility & $5(17.2 \%)$ \\
\hline Infertility etiologies & \\
\hline Tubal factors & $4(13.8 \%)$ \\
\hline Endometriosis & $2(6.9)$ \\
\hline Sperm Factors & $12(41.4 \%)$ \\
\hline Unexplained infertility & $11(37.9 \%)$ \\
\hline Recurrent IUl failure & $7(24.1 \%)$ \\
\hline Clinical characteristics & \\
\hline Anti Müllerian hormones $(\mathrm{ng} / \mathrm{mL})$ & $2.9 \pm 3.32$ \\
\hline Antral follicle count & $2.94(0.13-6.70)$ \\
\hline Basal Follicle-stimulating hormone $(\mathrm{mlU} / \mathrm{mL})$ & $7.15 \pm 1.60$ \\
\hline Basal Luteinizing hormone $(\mathrm{mlU} / \mathrm{mL})$ & $6(0.1-15.1)$ \\
\hline Basal Estradiol (pg/mL) & $36.50 \pm 9.32$ \\
\hline Basal progesterone $(\mathrm{ng} / \mathrm{mL})$ & $0.14(0.05-5.97)$ \\
\hline Total gonadotropin usage $(\mathrm{IU})$ & $2.025(1.050-3.000)$ \\
\hline Estradiol on the trigger day $(\mathrm{pg} / \mathrm{mL})$ & $2.212(1.169-5.855)$ \\
\hline Progesterone on the trigger day $(\mathrm{ng} / \mathrm{mL})$ & $0.55(0.20-1.35)$ \\
\hline Endometrial thickness $(\mathrm{mm})$ & $12.09(8-16)$ \\
\hline Embryology results & \\
\hline Number of mature oocytes & \\
\hline Number of top-quality blastocysts & \\
\hline
\end{tabular}




\begin{tabular}{|ll|}
\hline Parameters & Value $(\mathbf{n}=\mathbf{2 9})$ \\
\hline Number of embryo transfers & $1(1-2)$ \\
\hline
\end{tabular}

Bivariate analysis results in Table 2 showed a statistically significant difference in the $\mathrm{VI}$ and $\mathrm{VFI}$ value on the trigger day ( $p$ value $<0.05$ ) between the pregnant and non-pregnant women, respectively. The same trend was also observed on the day of OPU in which the VI and VFI values of pregnant women were significantly higher in comparison to the non-pregnant group ( $p$-value $<0.05$, respectively). On the other hand, no significant differences were observed in the overall endometrial vascularization parameters assessed on the day of embryo transfer ( $p$-value $>0.05$ ). Furthermore, the expression of LIF protein in pregnant women was significantly higher than the non-pregnant women ( $p$ value $=0.003$ ). 
Table 2

Bivariate analysis of three-dimensional power Doppler angiography ultrasound and LIF protein concentration.

\begin{tabular}{|c|c|c|c|c|c|}
\hline $\begin{array}{l}\text { Day of } \\
\text { assessment }\end{array}$ & Parameters & $\begin{array}{l}\text { Pregnant women } \\
(n=11)\end{array}$ & $\begin{array}{l}\text { Non-pregnant women } \\
(n=18)\end{array}$ & $p$-value & $\begin{array}{l}\text { Mean } \\
\text { Difference }\end{array}$ \\
\hline \multirow[t]{3}{*}{ Trigger day } & VI & $8.02 \pm 3.83$ & $4.67 \pm 5.92$ & $0.020 *$ & $\begin{array}{l}0.88(0.09- \\
1.67)\end{array}$ \\
\hline & $\mathrm{FI}$ & $28.93 \pm 1.78$ & $27.62 \pm 3.61$ & 0.274 & $\begin{array}{l}1.30 \\
(-1.09- \\
3.71)\end{array}$ \\
\hline & VFI & $2.38 \pm 1.15$ & $1.40 \pm 1.94$ & $0.020 *$ & $\begin{array}{l}0.49(0.03- \\
0.94)\end{array}$ \\
\hline \multirow[t]{3}{*}{ Ovum pick up } & VI & $4.08 \pm 3.06$ & $1.88 \pm 2.20$ & $0.028^{\star}$ & $\begin{array}{l}0.68(0.07- \\
1.29)\end{array}$ \\
\hline & $\mathrm{FI}$ & $29.36 \pm 4.60$ & $28.01 \pm 5.48$ & 0.466 & $\begin{array}{l}0.13 \\
(-0.23- \\
0.49)\end{array}$ \\
\hline & VFI & $1.31 \pm 1.08$ & $0.61 \pm 0.83$ & $0.038^{\star}$ & $\begin{array}{l}0.39(0.02- \\
0.76)\end{array}$ \\
\hline \multirow[t]{3}{*}{$\begin{array}{l}\text { Embryo } \\
\text { transfer }\end{array}$} & VI & $2.95 \pm 1.80$ & $2.29 \pm 2.52$ & 0.231 & $\begin{array}{l}0.35 \\
(-0.23- \\
0.93)\end{array}$ \\
\hline & $\mathrm{FI}$ & $29.58 \pm 4.24$ & $28.28 \pm 4.84$ & 0.619 & $\begin{array}{l}1.30 \\
(-2.33- \\
4.93)\end{array}$ \\
\hline & VFI & $0.95 \pm 0.68$ & $0.76 \pm 0.90$ & 0.282 & $\begin{array}{l}0.19 \\
(-0.16- \\
0.55)\end{array}$ \\
\hline $\begin{array}{l}\text { Embryo } \\
\text { transfer }\end{array}$ & $\begin{array}{l}\text { LIF } \\
(\mathrm{pg} / \mathrm{mL})\end{array}$ & $1200 \pm 376$ & $643 \pm 636$ & $0.003^{\star}$ & $\begin{array}{l}10.91 \\
(4.06- \\
17.76)\end{array}$ \\
\hline
\end{tabular}

Multiple logistic regression analysis was performed to control the confounding factors which include female age, antral follicle count, basal LH, number of mature oocytes, and endometrial thickness. After adjusting for the confounders, only $\mathrm{VI}$ on the trigger day and LIF protein concentration remains to be significantly different between pregnant and non-pregnant women ( $p$ value $=0.041$, Adjusted OR 2.593, $95 \% \mathrm{Cl} 1.03-6.47$ and $p$ value $=0.006$, Adjusted OR 1.196, 95\% Cl 1.05-1.35, respectively).

Receiver operating curve (ROC) analyses were presented in Table 3. The area under the curve of each parameter has a good predictive value for clinical pregnancy prediction (Fig. 1). Optimal cut off point of LIF protein was $>703.5 \mathrm{pg} / \mathrm{mL}(p$-value $<0.001)$ with $73.3 \%$ positive predictive value (PPV) and $100 \%$ 
negative predictive value (NPV). VI index variable on the trigger day also provided a sufficient predictive value to predict clinical pregnancy with a cut-off value of $>5.381$ ( $p$-value 0.003 ).

Table 3

$\mathrm{ROC}$ analysis of $\mathrm{VI}$ and LIF protein

\begin{tabular}{|lllllll|}
\hline Parameters & Sensitivity & Specificity & AUC & Standard error & $p$ value & $95 \% \mathrm{Cl}$ \\
\hline $\mathrm{VI}$ & $73 \%$ & $83 \%$ & 0.803 & 0.085 & $0.003^{*}$ & $0.636-0.970$ \\
LIF protein & $82 \%$ & $89 \%$ & 0.884 & 0.074 & $<0.001^{*}$ & $0.074-1.000$ \\
\hline
\end{tabular}

*Statistically significant; AUC: area under the curve; Vl: Vascularization Index; LIF: Leukemia inhibitory factor.

The potential synergistic role of VI on the HCG day and the expression of LIF protein in the prediction of endometrial receptivity of IVF cycles was formulated utilizing the cut-off value. The algorithm presented in Table 4 was sufficient to differentiate pregnant and non-pregnant cases ( $p$-value $<0.001$, sensitivity $81.8 \%$, specificity $94.44 \%$ ). The positive predictive value (PPV) and the negative predictive value (NPN) values were $90.0 \%$ and $89.47 \%$, respectively.

Table 4

Algorithm for the classification of pregnancy by combining the cut-off value of VI and LIF.

\begin{tabular}{|llll|}
\hline Classification & LIF protein in the implantation window $(\mathrm{pg} / \mathrm{mL})$ & IV on the HCG day & $\mathbf{N}(\%)$ \\
\hline Pregnant & $>703,5$ & $>5,381$ & $10(34.5 \%)$ \\
\hline \multirow{2}{*}{ Non-pregnant } & $>703,5$ & $<5,381$ & $19(65.5 \%)$ \\
\cline { 2 - 4 } & $<703,5$ & $>5,381$ & \\
\hline & $<703,5$ & $<5,381$ & \\
\hline
\end{tabular}

\section{Discussion}

The present study has demonstrated the good predictive potential of sub-endometrial VI index measurement on the maturation trigger day and LIF protein concentration in the implantation window for predicting endometrial receptivity that is characterized by successful implantation. Although the use of power Doppler indices as a marker has produced mixed findings [2], this study has displayed the favorable capacity of sub-endometrial VI index on the trigger day as an indicator for the likelihood of pregnancy. Considering the ever-changing process of sub-endometrial vascularization which is dynamically influenced by the estradiol and progesterone hormones [18, 19], serial assessments of the respective markers were obtained starting from the day of maturation trigger, ovum pick-up, and at the window of implantation of the fresh IVF cycle, while consecutive measurement in the frozen cycle was conducted by Nandi and Coworkers [19]. 
After performing multiple logistic regression analyses, our results were similar to the previous finding [2, 6] in regard to the optimal time point of sub-endometrial measurement. $\mathrm{Ng}$ and colleagues [6] demonstrated that the overall sub-endometrial indices assessed on the day of oocyte retrieval were not predictive of the pregnancy event. Likewise, a recent meta-analysis also failed to detect the significance of Doppler signal indices on the day of embryo transfer to differentiate women who achieved clinical pregnancy and women who did not [2]. Interestingly, the present study confirms that the measurement of sub-endometrial $\mathrm{VI}$ on the day of maturation trigger could provide useful information among other time points in the fresh IVF cycle. Using different ultrasonography and Doppler parameters such as enddiastolic blood flow, endometrial pattern, and endometrial thickness, our results have supported the previous findings conducted by Dechaud and Colleagues [20], which established that the prominent information to predict the likelihood of pregnancy is attained when those parameters were measured on the day of trigger maturation rather than on the day of oocyte retrieval and embryo transfer.

On the contrary, a limited power of using sub-endometrial $\mathrm{VI}$ on the day of maturation trigger for pregnancy prediction was reported by $\mathrm{Ng}$ and Coworkers in another study [7]. Nevertheless, the study design and the ovarian stimulation protocol were different from our study. While that study employed a long protocol for the IVF stimulation, our study recruited women who were subjected to the antagonist protocol. These differences might complicate the comparison of the study results. Another study also observed that the VFI index on the trigger day was highly predictive of pregnancy rather than the $\mathrm{VI}$ and $\mathrm{FI}$ indices which contradict our finding [21]. On the other hand, endometrial VI, FI, and VFI were shown to be significantly higher in the pregnant group than those of the non-pregnant group [4, 5]. However, this study could not confirm those results since the areas of measurement were different.

The result of our study has also supported the expanding and consistent claims of LIF as a good marker to predict endometrial receptivity $[8,11,12,22]$. Either glandular or luminal epithelial cells of the endometrium and also the blastocyst are known to express LIF and LIF receptors (LIF-R and gp130). Moreover, LIF expression in endometrium increases after ovulation day and attains its optimum concentration at the implantation window, suggesting the indispensable role of LIF and its receptors in the event of implantation. Simultaneously with a plethora of other cytokines, LIF is proposed to regulate the migration and invasion capacity of extra villous trophoblast on the maternal site. Once the syncytiotrophoblast has successfully invaded the luminal epithelial cells, the blastocyst starts to produce its own cytokines such as LIF and interleukin 1, which subsequently induce the increased expression of LIF in the endometrium. The crosstalk through cytokines is believed to be a remarkable mechanism that promotes the completion of implantation [23].

The successful implantation of LIF ${ }^{-} /^{-}$mice blastocyst in the pseudo pregnant mice demonstrated the prominent role of the endometrial LIF rather than the embryo-secreted LIF. In humans, endometrial LIF expression level can be measured by utilizing different sampling methods including uterine secrete aspiration [17], flushing [11, 24], or biopsy [11, 25]. In a prospective follow-up study, Mikolajczyk and Coworkers [24] collected uterine flushing samples prior to laparoscopy for LIF measurements in infertile women diagnosed with minimal grade endometriosis (stage I-II), luteal phase defect, and idiopathic 
infertility. After eighteen months of receiving fertility treatments, questionnaires were sent out to all studied participants to follow up with the treatment outcomes. It was found that the infertile women who had failed to achieve natural pregnancy had a lower LIF expression than the women who had been pregnant $(1.1 \mathrm{pg} / \mathrm{mL}$ Vs. $22.07 \mathrm{pg} / \mathrm{mL} ; p$ value $<0.008)$. While the mentioned study suggested LIF as a predictor for the naturally conceived live-birth, our study has indicated its additional potential role in predicting the clinical pregnancy of an ovarian stimulation IVF program. Furthermore, the endometrial LIF expression presented in this study was closely related to the actual environmental condition of the uterus just before the embryo transfer procedure.

The strength of our study is in the presented results which suggest the availability of a reliable, quick, and simple alternative method to measure endometrial receptivity by simply aspirating the uterine secretion sample at the implantation window for clinical practice in the IVF program. We supported the results of previous studies that a small amount of endometrial secretion could be gently aspirated for the analysis of the various implantation biomarkers without reducing the chance of pregnancy $[16,17]$. This current study has confirmed the safety and effectiveness of the endometrial secretion aspiration method for pregnancy prediction. Furthermore, as the gold standard of measuring endometrial receptivity relies immensely on the implantation event, by carefully selecting the good prognosis subjects who had successfully obtained good quality blastocyst for transfer, this study has reduced the potential bias that might arise from complications of the embryo.

This study also pertains to a limitation in which the number of participants is relatively small but, nonetheless, statistically adequate to prove the clinical use of sub-endometrial VI index and LIF as indicators for endometrial receptivity. Furthermore, this study has yet to discover the in-depth association between the VI index and the expression of LIF during the implantation window. Notably, a concordance decreased of VI index on the trigger day and low expression of LIF were more apparent in the nonpregnant group compared to the pregnant group. Further attempt to explore the relationship between $\mathrm{VI}$ index and LIF expression during the implantation window is worth pursuing in the interest of gaining a broader perspective on the pathophysiology of aberrant LIF secretion that leads to impaired implantation.

In conclusion, measuring the sub-endometrial VI index on the day of maturation trigger is most appropriate for a pregnancy prediction rather than on the day of oocyte retrieval and embryo transfer. Endometrial LIF expression at the window of implantation also poses as a potential predictor for pregnancy in IVF. A combination of the cut-off values of VI and LIF also yielded a simple noteworthy algorithm for the prediction of pregnancy.

\section{Declarations}

\section{Research Funding:}

The author received no specific funding to conduct the study.

\section{Competing of Interest:}


The authors declared that they have no conflict of interest or competing interests to disclose. This study was conducted as part of doctoral degree dissertation of author IS.

\section{Availability of data and material:}

Data is available upon request

\section{Ethical Approval:}

This study was performed in line with the principles of the Declaration of Helsinki. The study protocol has been reviewed and approved by the Ethical Committee, Faculty of Medicine, University of Indonesia (Number of ethic approval: 0699/UN2.F1/ETIK/2018).

\section{Informed Consent:}

All subjects have given their signed informed written consent to participate in this study.

\section{References}

1. Lessey BA, Young SL. What exactly is endometrial receptivity? Fertil Steril [Internet]. 2019;111(4):611-7. Available from: https://doi.org/10.1016/j.fertnstert.2019.02.009

2. Craciunas L, Gallos I, Chu J, Bourne T, Quenby S, Brosens JJ et al (2019) Conventional and modern markers of endometrial receptivity: A systematic review and meta-analysis. Hum Reprod Update 25(2):202-223

3. Kupesic $S$, Kurjak A. Uterine and ovarian perfusion during the periovulatory period assessed by transvaginal color Doppler. Fertil Steril [Internet]. 1993;60(3):439-43. Available from: http://dx.doi.org/10.1016/S0015-0282(16)56157-3

4. Elsokkary M, Eldin AB, Abdelhafez M, Rateb A, Samy M, Eldorf A et al. The reproducibility of the novel utilization of five-dimensional ultrasound and power Doppler in the prediction of endometrial receptivity in intracytoplasmic sperm-injected women: a pilot prospective clinical study. Arch Gynecol Obstet [Internet]. 2019;299(2):551-8. Available from: https://doi.org/10.1007/s00404-018-5001-4

5. Mercé LT, Barco MJ, Bau S, Troyano J (2008) Are endometrial parameters by three-dimensional ultrasound and power Doppler angiography related to in vitro fertilization/embryo transfer outcome? Fertil Steril 89(1):111-117

6. Ng EHY, Chan CCW, Tang OS, Yeung WSB, Ho PC (2006) The role of endometrial and subendometrial blood flows measured by three-dimensional power Doppler ultrasound in the prediction of pregnancy during IVF treatment. Hum Reprod 21(1):164-170

7. Ng EHY, Chan CCW, Tang OS, Yeung WSB, Ho PC. Changes in endometrial and subendometrial blood flow in IVF. Reprod Biomed Online [Internet]. 2009;18(2):269-75. Available from: http://dx.doi.org/10.1016/S1472-6483(10)60265-9 
8. Kondera-Anasz Z, Sikora J, Mielczarek-Palacz A (2004) Leukemia inhibitory factor: An important regulator of endometrial function. Am J Reprod Immunol 52(2):97-105

9. Singh M, Chaudhry P, Asselin E (2011) Bridging endometrial receptivity and implantation: Network of hormones, cytokines, and growth factors. J Endocrinol 210(1):5-14

10. Stewart CL, Kaspar L, Brunet LJ, Bhatt H, Gadi I, Kontgen F et al (1992) Blastocyst implantation depends on maternal expression of leukaemia inhibotory factors. Nature 359:76-79

11. Tawfeek MA, Eid MA, Hasan AM, Mostafa M, El-Serogy HA. Assessment of leukemia inhibitory factor and glycoprotein 130 expression in endometrium and uterine flushing: a possible diagnostic tool for impaired fertility. BMC Womens Health. 2012;12

12. Pathare ADS, Zaveri K, Hinduja I (2017) Downregulation of genes related to immune and inflammatory response in IVF implantation failure cases under controlled ovarian stimulation. Am J Reprod Immunol 78(1):1-13

13. Charnock-Jones DS, Sharkey AM, Fenwick P, Smith SK (1994) Endometrium At the Time of Implantation and the Blastocyst Contains. J Reprod Fertil 101:421-426

14. Laird SM, Tuckerman EM, Dalton CF, Dunphy BC, Li TC, Zhang X (1997) The production of leukaemia inhibitory factor by human endometrium: Presence in uterine flushings and production by cells in culture. Hum Reprod 12(3):569-574

15. Gardner DK, Lane M, Stevens J, Schlenker T, Schoolcraft WB (2000) Blastocyst score affects implantation and pregnancy outcome: Towards a single blastocyst transfer. Fertil Steril 73(6):11551158

16. van der Gaast MH, Beier-Hellwig K, Fauser BCJM, Beier HM, Macklon NS. Endometrial secretion aspiration prior to embryo transfer does not reduce implantation rates. Reprod Biomed Online [Internet]. 2003;7(1):105-9. Available from: http://dx.doi.org/10.1016/S1472-6483(10)61737-3

17. Boomsma CM, Kavelaars A, Eijkemans MJC, Amarouchi K, Teklenburg G, Gutknecht D et al. Cytokine profiling in endometrial secretions: A non-invasive window on endometrial receptivity. Reprod Biomed Online [Internet]. 2009;18(1):85-94. Available from: http://dx.doi.org/10.1016/S14726483(10)60429-4

18. Raine-Fenning NJ, Campbell BK, Kendall NR, Clewes JS, Jonhson IR (2004) Quantifying the changes in endometrial vascularity throughout the normal menstrual cycle with three-dimensional power Doppler angiography. Hum Reprod 19(2):330-338

19. Nandi A, Martins WP, Jayaprakasan K, Clewes JS, Campbell BK, Raine-Fenning NJ. Assessment of endometrial and subendometrial blood flow in women undergoing frozen embryo transfer cycles. Reprod Biomed Online [Internet]. 2014;28(3):343-51. Available from: http://dx.doi.org/10.1016/j.rbmo.2013.11.004

20. Dechaud H, Bessueille E, Bousquet P, Reyftmann L, Hamamah S, Hedon B (2008) Optimal timing of ultrasonographic and Doppler evaluation of uterine receptivity to implantation. Reprod Biomed Online 16(3):368-375 
21. Wu HM, Chiang CH, Huang HY, Chao AS, Wang HS, Soong YK (2003) Detection of the subendometrial vascularization flow index by three-dimensional ultrasound may be useful for predicting the pregnancy rate for patients undergoing in vitro fertilization-embryo transfer. Fertil Steril 79(3):507511

22. Ledee-Ba- taille N, Frydman FO, Taupin R, Kadoch J, Chaouat J G. Concentration of leukemia inhibitory factor (LIF) in uterine flushing fluid is highly predictive of embryo implantation, S110 Abstracts S111. 2002;74(3):110-1

23. Fitzgerald JS, Poehlmann TG, Schleussner E, Markert UR (2008) Trophoblast invasion: The role of intracellular cytokine signalling via signal transducer and activator of transcription 3 (STAT3). Hum Reprod Update 14(4):335-344

24. Mikolajczyk M, Wirstlein P, Skrzypczak J (2007) The impact of leukemia inhibitory factor in uterine flushing on the reproductive potential of infertile women - A prospective study. Am J Reprod Immunol 58(1):65-74

25. Hasegawa E, Ito H, Hasegawa F, Hatano K, Kazuka M, Usuda S et al. Expression of leukemia inhibitory factor in the endometrium in abnormal uterine cavities during the implantation window. Fertil Steril [Internet]. 2012;97(4):953-8. Available from: http://dx.doi.org/10.1016/j.fertnstert.2012.01.113

\section{Figures}




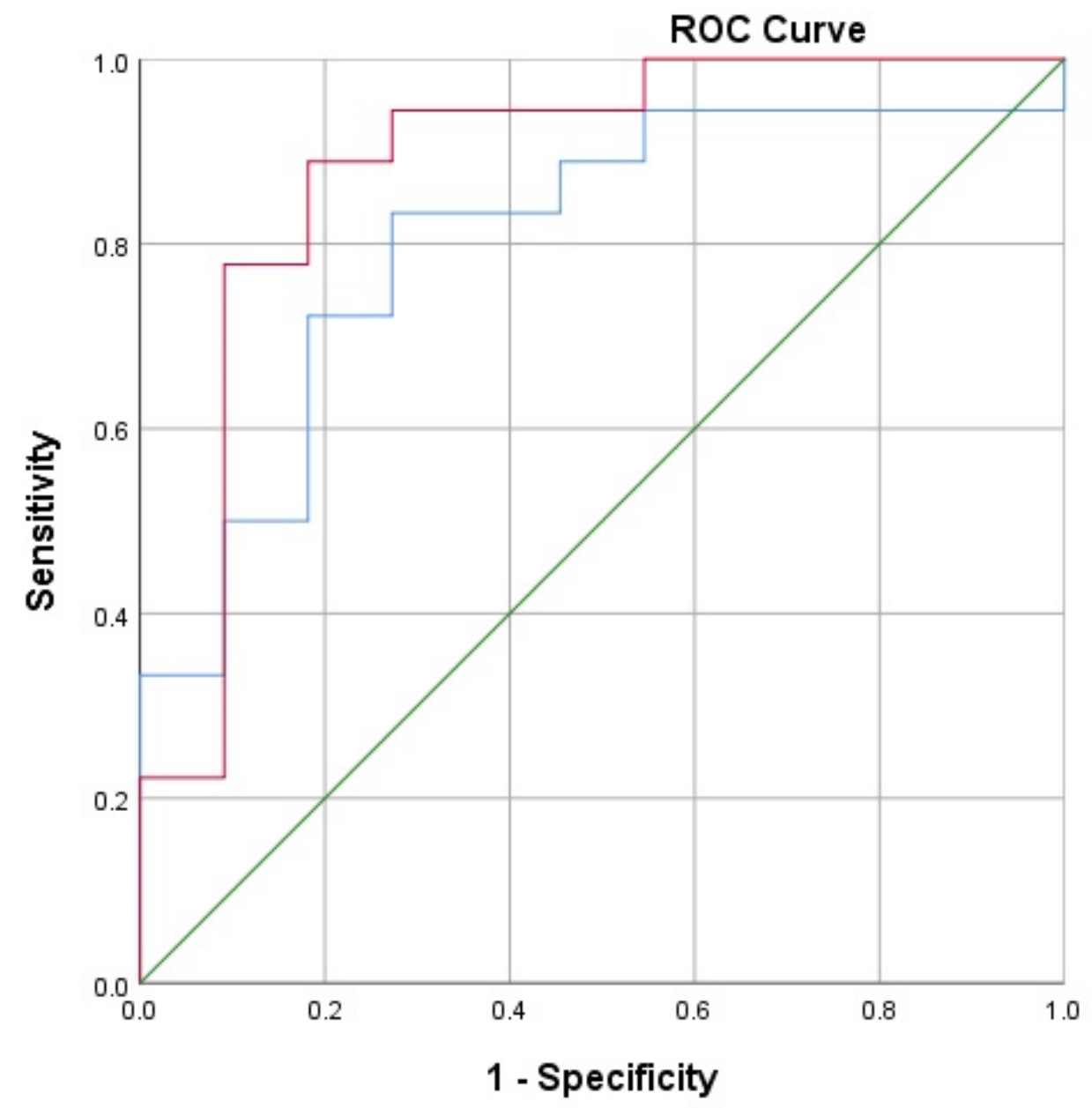

Figure 1

ROC curve for VI on the trigger maturation day and LIF in predicting endometrial receptivity. Red line: LIF; Blue line: Vl; Green line: reference. 